\title{
Identification of Four Pathological Stage-Relevant Genes in Association with Progression and Prognosis in Clear Cell Renal Cell Carcinoma by Integrated Bioinformatics Analysis
}

\author{
Dengyong Xu, ${ }^{1}$ Yuzi Xu, ${ }^{2,3}$ Yiming Lv, ${ }^{1}$ Fei Wu, ${ }^{4}$ Yunlong Liu, ${ }^{5}$ Ming Zhu, ${ }^{6}$ Dake Chen $\left(\mathbb{D},{ }^{7}\right.$ \\ and Bingjun Bai ${ }^{1}{ }^{1}$ \\ ${ }^{1}$ Department of Colorectal Surgery, Sir Run Run Shaw Hospital, Zhejiang University School of Medicine, Hangzhou 310016, China \\ ${ }^{2}$ Department of Oral Implantology and Prosthodontics, The Affiliated Stomatology Hospital, Zhejiang University School of Medicine, \\ Hangzhou 310006, China \\ ${ }^{3}$ Key Laboratory of Oral Biomedical Research of Zhejiang Province, Zhejiang University School of Stomatology, \\ Hangzhou 310006, China \\ ${ }^{4}$ School of Medicine, Anhui University of Science and Technology, Huainan 232001, China \\ ${ }^{5}$ Department of Medical Oncology, Sir Run Run Shaw Hospital, Zhejiang University School of Medicine, Hangzhou 310016, China \\ ${ }^{6}$ Department of Nephrology, The First People's Hospital of Huzhou, The First Affiliated Hospital of Huzhou Teachers College, \\ Huzhou, Zhejiang, China \\ ${ }^{7}$ Department of Urology, Wenzhou People's Hospital, Wenzhou, Zhejiang, China
}

Correspondence should be addressed to Dake Chen; 61502485@qq.com and Bingjun Bai; echobai@zju.edu.cn

Received 15 December 2019; Accepted 9 March 2020; Published 28 March 2020

Academic Editor: Rui Liu

Copyright ( 2020 Dengyong Xu et al. This is an open access article distributed under the Creative Commons Attribution License, which permits unrestricted use, distribution, and reproduction in any medium, provided the original work is properly cited.

Clear cell renal cell carcinoma (ccRCC) is a major histological subtype of renal cell carcinoma and can be clinically divided into four stages according to the TNM criteria. Identifying clinical stage-related genes is beneficial for improving the early diagnosis and prognosis of ccRCC. By using bioinformatics analysis, we aim to identify clinical stage-relevant genes that are significantly associated with the development of ccRCC. First, we analyzed the gene expression microarray data sets: GSE53757 and GSE73731. We divided these data into five groups by staging information-normal tissue and ccRCC stages I, II, III, and IV-and eventually identified 500 differentially expressed genes (DEGs). To obtain precise stage-relevant genes, we subsequently applied weighted gene coexpression network analysis (WGCNA) to the GSE73731 dataset and KIRC data from The Cancer Genome Atlas (TCGA). Two modules from each dataset were identified to be related to the tumor TNM stage. Several genes with high inner connection inside the modules were considered hub genes. The intersection results between hub genes of key modules and 500 DEGs revealed UBE2C, BUB1B, RRM2, and TPX2 as highly associated with the stage of ccRCC. In addition, the candidate genes were validated at both the RNA expression level and the protein level. Survival analysis also showed that 4 genes were significantly correlated with overall survival. In conclusion, our study affords a deeper understanding of the molecular mechanisms associated with the development of ccRCC and provides potential biomarkers for early diagnosis and individualized treatment for patients at different stages of ccRCC.

\section{Introduction}

Renal cancer is the deadliest urinary malignancy, with more than 350,000 cases worldwide [1]. Each year, over 140,000 people die from renal cancer, and the disease still has an increasing incidence [2]. Clear cell renal cell carcinoma
(ccRCC), as the most common histologic subtype of renal cancer, can be clinically divided into four stages according to tumor size and the extent of invasion and metastasis $[3,4]$. Currently, radiotherapy and chemotherapy are largely ineffective in the treatment of ccRCC, so surgery is the main treatment for most ccRCC, especially at the early stage $[5,6]$. 
Unfortunately, most of the patients do not present any specific signs, and only $30 \%$ can be diagnosed during the early stage $[7,8]$. For patients progressing to advanced stages, targeted therapies have been proposed as the most potential nonsurgical treatments because of their specificity and low toxicity [9]. Many targeted drugs have been approved for clinical use, while many others are undergoing clinical trials [10]. Immune checkpoint inhibitors with or without combination with tyrosine kinase inhibitors are the current standard of care. However, the median survival time of the treated patients still remains at a low level [11], which is far from satisfactory. Therefore, to improve the rate of early diagnosis and prognosis of ccRCC, it is necessary to comprehensively study the tumorigenesis and clinical stages of ccRCC and establish a relationship with more novel and specific biomarkers.

Originating from the proximal tubule, ccRCC showed abundant clear cytoplasm under the microscope because of deposition of lipid and glycogen, especially for larger tumors [12]. Although smoking [13], hypertension [14], and obesity [15] are considered risk factors, genetic variation also plays a critical role during the tumorigenesis process. Some specific gene mutations and corresponding signal pathways have already been proven to be closely associated with ccRCC [16]. Nearly $90 \%$ of ccRCC is characterized by the aberration of VHL [17], while PBRM1 is considered the second major tumor suppressor gene in ccRCC [18]. Previous studies have revealed a correlation between the lower expression of VHL and PBRM1 and a higher Fuhrman grade [19]. BAP1 is another tumor suppressor in ccRCC $[20,21]$, the low expression of which is significantly associated with high grade but not survival [22]. However, another study has indicated that loss of BAP1 expression suggests poor prognosis in metastatic ccRCC [23]. Therefore, dynamic changes in genes in different stages are of great importance in the occurrence and development of ccRCC, as well as the treatment and prognosis of this disease. Notably, a great difference remains in prognosis depending on whether the disease is diagnosed earlier or later. The 5-year overall survival rate is $92 \%$ if diagnosed in stage I but drops sharply to $23 \%$ in stage IV. Thus, identifying clinical stage-related genes is beneficial for improving the early diagnosis and prognosis of ccRCC.

Currently, bioinformatics analysis is becoming a useful approach to identify relevant genes to certain diseases. Weighted gene coexpression network analysis (WGCNA) [24] has emerged as an effective method for analyzing gene expression data and to discover the relationship between gene clusters and tumor phenotypes. Several researchers have applied this approach to screen the genes involved in the genesis of ccRCC [25-29]. They take the understanding of the molecular mechanisms of ccRCC a step further. However, precise and efficacious molecular targets for the treatments of ccRCC have not been found. Thus, identifying novel therapeutic targets or biomarkers is still a priority for diagnostic or prognostic applications.

In this study, we aim to more precisely identify clinical stage-related differentially expressed genes (DEGs) that are significantly associated with the occurrence and development of ccRCC by applying integrated bioinformatics analysis. We analyzed a total of 261 raw data files from GSE53757 and GSE73731, then divided the data into five groups and compared the gene expression of normal tissue with ccRCC stages I, II, III, and IV. As a result, we identified 500 common DEGs that were either upregulated or downregulated in each stage of ccRCC. Furthermore, functional enrichment analyses were performed and protein-protein interaction (PPI) networks were constructed so as to explore the biological roles of those DEGs. Moreover, to obtain DEGs highly correlated with the stage of ccRCC, WGCNA was applied to detect the modules and hub genes associated with the tumor stage in two independent data sets: The Cancer Genome Atlas Kidney Renal Clear Cell Carcinoma (TCGA-KIRC) data and GSE73731. Finally, the intersection results between the hub genes from key modules and 500 DEGs showed UBE2C, BUB1B, RRM2, and TPX2 as key hub genes that were highly associated with the clinical stages of ccRCC. qPCR and the Human Protein Atlas database were utilized to validate the roles of key hub genes both at the RNA expression level and at the protein level. Survival analysis showed that these 4 genes were all significantly related to overall survival. In short, UBE2C, BUB1B, RRM2, and TPX2 could probably be potential biomarkers for early diagnosis and individualized treatment for ccRCC.

\section{Materials and Methods}

2.1. Screening and Preprocessing of Microarray Data. All data in this manuscript were collected under the guidelines approved by the First Affiliated Hospital of Zhejiang University School of Medicine's institutional review board and complied with the current laws in China. Neglecting language, race, region, and time constraints, we systematically researched the Gene Expression Omnibus (GEO) database (https://www.ncbi.nlm.nih.gov/geo/) with several defined strategies: (1) tissue source was ccRCC and/or paracancerous tissue, (2) the Affymetrix platform was GPL570, (3) data sets had staging information, and (4) the tissue came from patients who did not receive any antitumor treatments. The gene expression profiles of GSE53757 and GSE73731 were downloaded and analyzed by the affy package [30] in R software ( $\mathrm{R}$ version 3.6). Relative logarithmic expression (RLE) and normalized unscaled standard errors (NUSE) [31] were used to evaluate the quality of the data. We fitted a probelevel model to the data and created two plots for the dataset. The deviant arrays can be identified by their not being centered at 0 in the RLE boxplot or 1 in the RUSE boxplot or being more spread out than the other arrays. After excluding the outliers, we performed a standard robust multiarray average (RMA) [32] procedure to create an expression matrix. During the process, the raw intensity values were background-corrected, log2-transformed, and then quantile-normalized.

2.2. Identification of DEGs. Raw data files contained in GSE53757 and GSE73731 were divided into five groups according to their tissue source and stage: ccRCC stages I, II, III, and IV and normal tissues. The gene expression levels in ccRCC stages I, II, III, and IV were compared with those in 
normal tissue using the limma package [33] in R software. Those genes with the cut-off criteria of $|\log 2 \mathrm{FC}|>2$ (FC: fold change) and an adjusted $P$ value $<0.05$ [34] were considered DEGs, which were further divided into upregulated and downregulated genes.

2.3. Functional Enrichment Analysis. To explore the biological functional roles of DEGs associated with ccRCC, Gene Ontology (GO) and Kyoto Encyclopedia of Genes and Genomes (KEGG) pathway enrichment analyses were performed by using the clusterProfiler package [35] (http:// bioconductor.org/packages/release/bioc/html/clusterProfiler .html) in $\mathrm{R}$ software. Under the condition of $P<0.05$ and $q<0.02$, significant biological process (BP), molecular function (MF), cellular component (CC) terms, and KEGG pathways were selected and visualized.

2.4. PPI Network Construction. The online database STRING [36] (version 11; https://string-db.org/) performed PPI networks of DEGs, which covered a total of 9,643,763 proteins from 2,031 organisms. DEGs were uploaded to STRING, and the results were visualized in Cytoscape software [37] (version 3.7.0; http://www.cytoscape.org/) with the minimum required interaction score of 0.700 . In Cytoscape, dysregulated genes were plotted in different colors.

2.5. Data Collection and Preprocessing for WGCNA. Raw RNA sequence data and corresponding clinical information of KIRC patients were downloaded from TCGA. The data were concatenated to a matrix with gene symbols as row names and TCGA patient barcodes as column names. Then, we removed the control samples and samples with incomplete clinical information. Genes with zero counts in more than $80 \%$ of samples were also excluded. We normalized the matrix by the voom function [38] from the limma package [39], as RNA-seq read counts usually follow a negative binomial distribution. In addition to TCGA data, we also performed WGCNA on the normalized GSE73731 data. Finally, we chose the top $75 \%$ of the variant genes for WGCNA. In detail, the median absolute deviation (MAD) was used as a robust measure of variability.

2.6. Weighted Gene Coexpression Network Analysis. Weighted gene coexpression network analysis was performed using the WGCNA package in $\mathrm{R}$ [24]. The first step in this process was to calculate a similarity matrix using biweight midcorrelation [40], as it is more robust to outliers. After that, a weighted adjacency matrix was defined by raising the coexpression similarity to an appropriate softthresholding power. We chose the best power ( $\beta$-value) based on the criterion of approximate scale-free topology [41]. Then, to minimize the effects of noise and spurious associations, we transformed the adjacency into a topological overlap matrix (TOM) and calculated the corresponding dissimilarity [42]. We now used hierarchical clustering to produce a hierarchical clustering tree and dynamic tree cut method to assign coexpressed genes to each module [43]. Modules were constructed with a minimum module size of 30 genes, and highly similar modules were combined using a dissimilarity threshold of 0.25 .
TABLE 1: Real-time PCR primers.

\begin{tabular}{|c|c|}
\hline Gene & Real-time PCR primer sequences \\
\hline \multirow{2}{*}{ UBE2C } & F: 5' - AGTGGCTACCCTTACAATGCG -3' \\
\hline & R: 5' - TTACCCTGGGTGTCCACGTT - $3^{\prime}$ \\
\hline \multirow{2}{*}{ BUB1B } & F: 5' - AAATGACCCTCTGGATGTTTGG - $3^{\prime}$ \\
\hline & R: $5^{\prime}$ - GCATAAACGCCCTAATTTAAGCC - $3^{\prime}$ \\
\hline \multirow{2}{*}{ RRM2 } & F: $5^{\prime}$ - GTGGAGCGATTTAGCCAAGAA - $3^{\prime}$ \\
\hline & R: 5' - CACAAGGCATCGTTTCAATGG -3' \\
\hline \multirow{2}{*}{ TPX2 } & F: 5' - ATGGAACTGGAGGGCTTTTTC - $3^{\prime}$ \\
\hline & R: $5^{\prime}$ - TGTTGTCAACTGGTTTCAAAGGT - $3^{\prime}$ \\
\hline \multirow{2}{*}{$\beta$-Actin } & F: 5' - ACTCTTCCAGCСTTCCTTCC - $3^{\prime}$ \\
\hline & R: 5' - CGTCATACTCCTGCTTGCTG - $3^{\prime}$ \\
\hline
\end{tabular}

2.7. Identification of Stage-Related Modules. Along with the module identification procedure, the module eigengenes were generated by principal component analysis (PCA). We used these module eigengenes and external clinical parameters to perform a module-trait relationship (MTR) analysis. The Pearson correlation coefficient and $P$ value were calculated in this process. A heat map was drawn to present the results so that we could easily identify which modules related to the tumor stage. We also measured gene significance based on the correlation of a gene expression profile with a sample trait, and the module significance was the average absolute value of the gene significance measure for all genes in a given module. A barplot of the module significance for all modules detected was drawn. The highest module had the strongest correlation with the clinical trait.

2.8. Module Preservation Analysis. Sometimes, the modules identified are not reproducible in another dataset with similar samples, which means the quality of the modules is poor. Module quality measures based on density and separability measures can be used to confirm that the reference modules are well defined [44]. To verify whether the modules we selected are preserved, we utilized the modulePreservation function in the WGCNA package to calculate module preservation statistics between GSE73731 and an independent data set, GSE53757.

2.9. Identification of Hub Genes. After the construction of the network and the identification of stage-related modules, we explored individual genes within the coexpression module. The hub genes were selected on the basis of the module membership and gene significance. Module membership is measured by the correlation between the profile of the gene and the eigengene of the module, which describes how closely related the gene is to the module. Criteria for selecting the hub genes were as follows: module membership $>0.8$ and gene significance $>0.2$. Finally, we obtained the intersection of these hub genes and DEGs for further analysis.

2.10. Patient and Tissue Sample Collection. This study was approved by the Sir Run Run Shaw Hospital and Zhejiang 


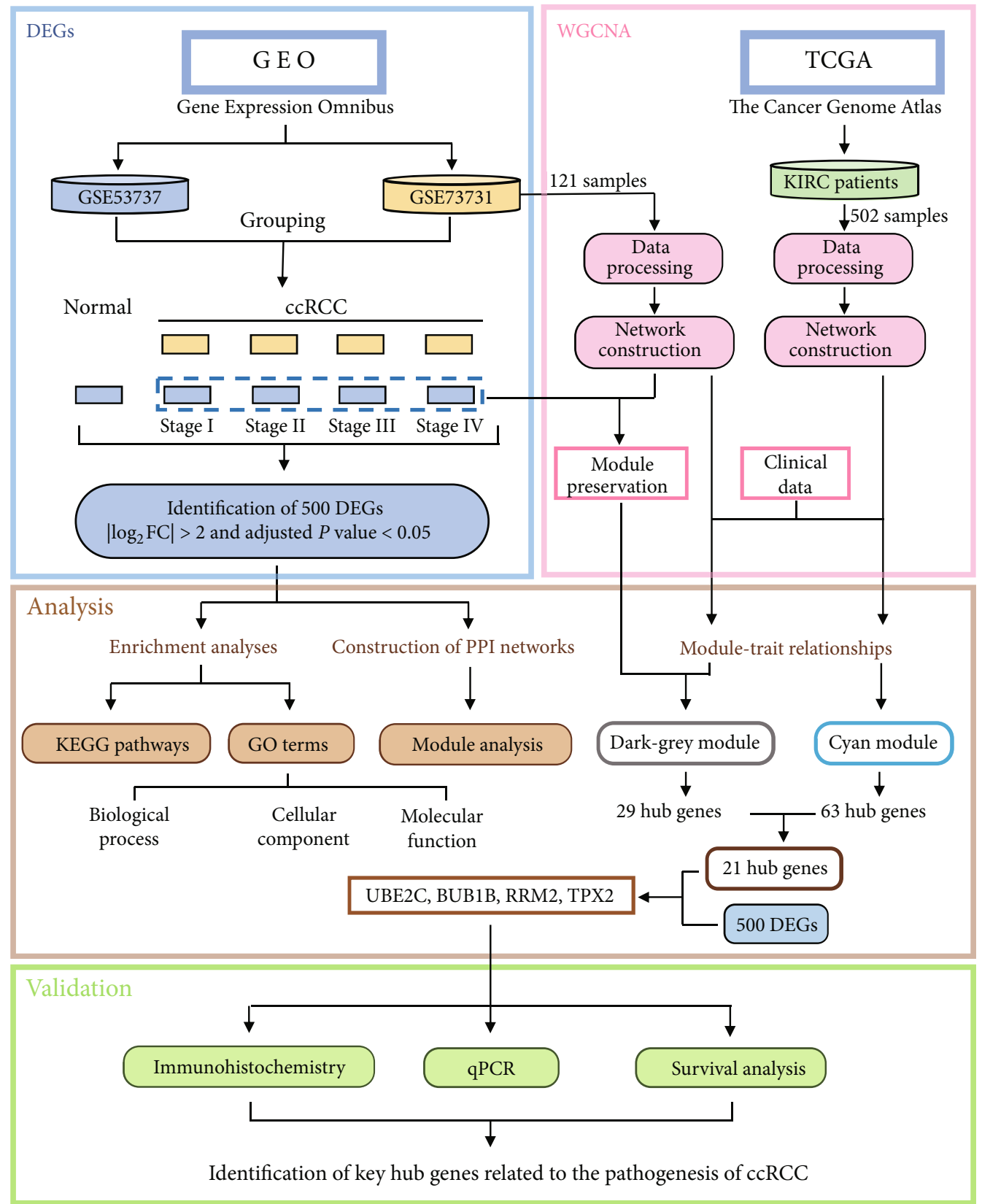

Figure 1: The flow chart of our study.

University Ethics Committee (No. 20100823). All patients involved in this study had informed consent. Fresh cancerous tissue samples were obtained directly from the operation specimens of 8 patients who had undergone surgical resections for the kidney at the Department of Urology, Sir Run Run Shaw Hospital, Hangzhou, Zhejiang, China, between September 2010 and March 2011. Written informed consent for tissue collection was obtained from all patients prior to their surgical procedures. The adjacent normal tissues were collected from more than $5 \mathrm{~cm}$ away from the cancerous tissue.

2.11. qPCR Validation of Key Hub Genes. The overlapping genes in hub genes and DEGs were defined as key hub genes.
TABLE 2: GSE datasets involved in our study.

\begin{tabular}{lcc}
\hline Group & Quantity & GSE datasets \\
\hline Normal tissues & 72 & GSE53757 \\
ccRCC stage I & 62 & GSE53757+GSE73731 \\
ccRCC stage II & 29 & GSE53757+GSE73731 \\
ccRCC stage III & 41 & GSE53757+GSE73731 \\
ccRCC stage IV & 57 & GSE53757+GSE73731 \\
Total & 261 & \\
\hline
\end{tabular}

Note: ccRCC: clear cell renal cell carcinoma; GEO: Gene Expression Omnibus; GSE: GEO series. 


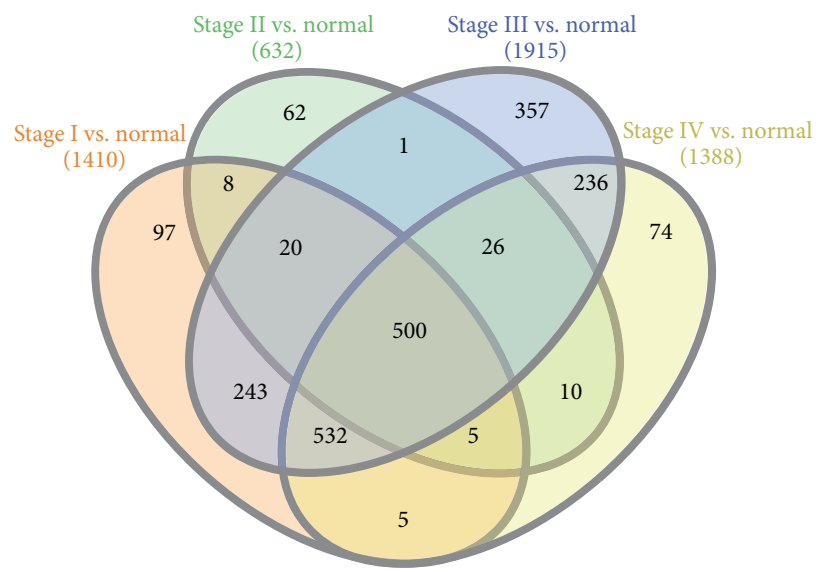

(a)

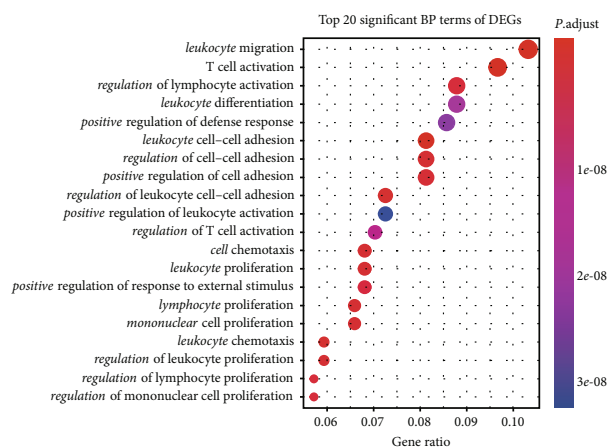

Count
$\mathbf{8}_{35}^{30}$$\quad \mathbf{Q}_{45}^{40}$

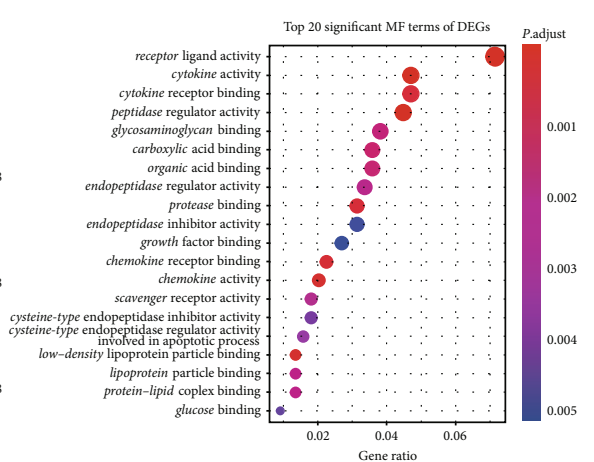

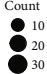

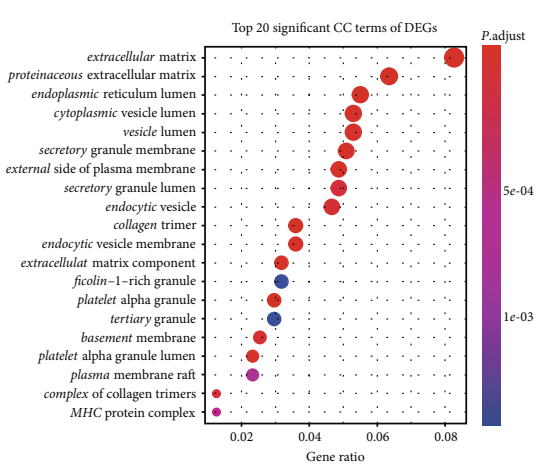

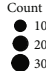

(b)

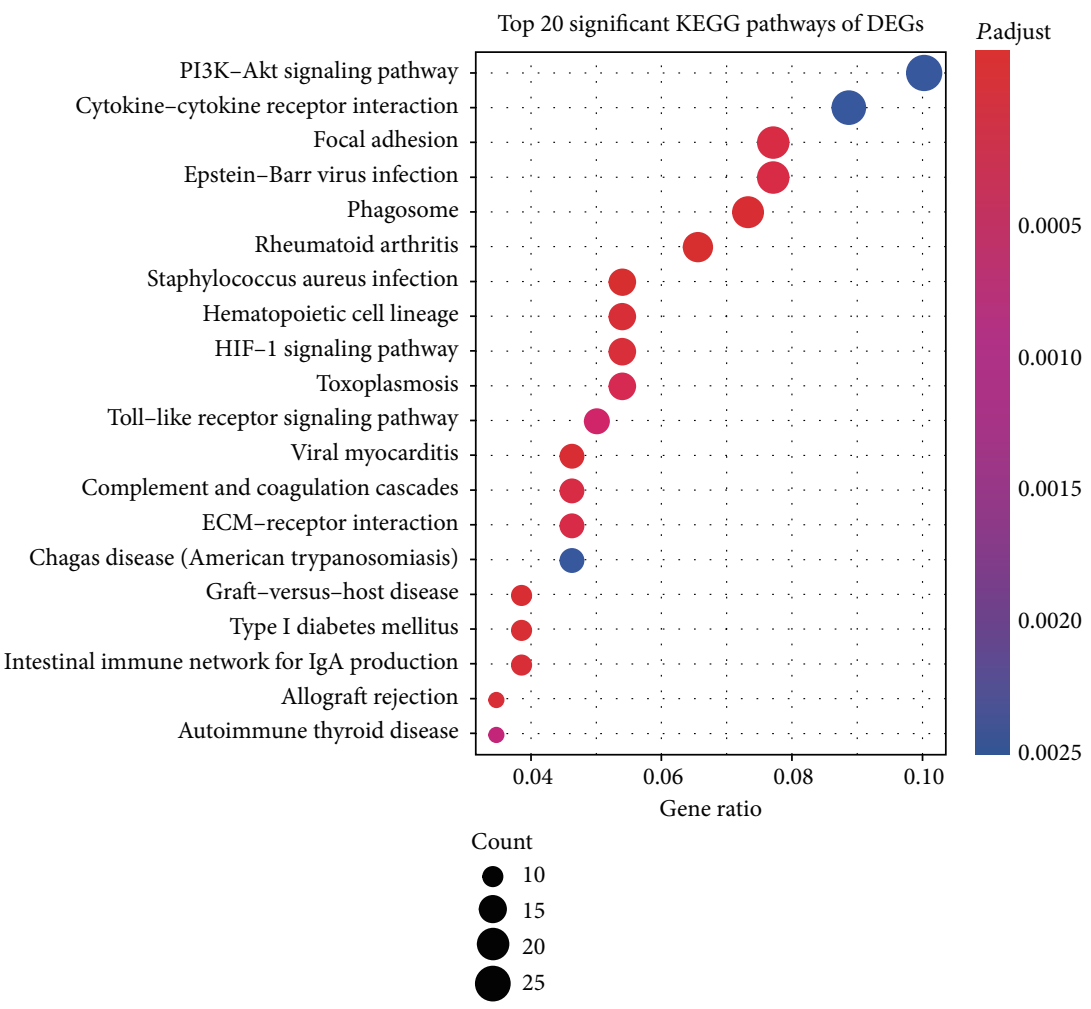

(c)

FIgURe 2: Continued. 


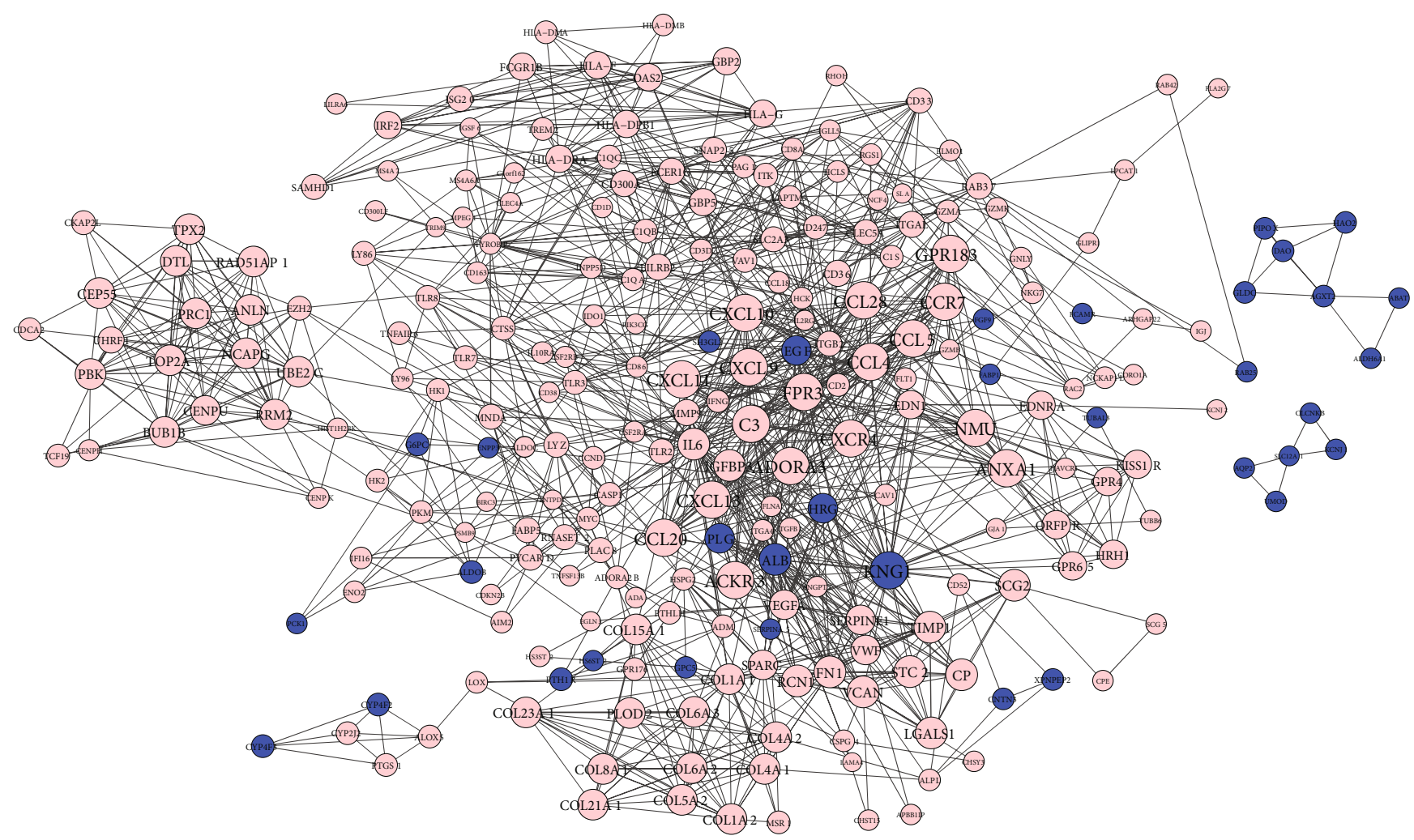

(d)

FIGURE 2: Identification and functional enrichment analyses of 500 DEGs. (a) Constructing a Venn diagram in four comparison groups with the cut-off criteria of $|\log 2 \mathrm{FC}|>2$ and an adjusted $P$ value $<0.05$. (b) GO enrichment analysis showing the top 20 terms of the BP, MF, and CC categories. (c) Top 20 significant KEGG pathways. (d) PPI networks of 500 DEGs. Pink nodes indicate upregulated DEGs and blue nodes indicate downregulated DEGs.

We validated their expression levels by qPCR. Following the manufacturer's instructions, the RNA of tissue samples was extracted using a TRIzol reagent (Invitrogen; Thermo Fisher Scientific, Inc., Waltham, MA, USA). RNA was quantified by using a NanoDrop 2000c spectrophotometer (Thermo Fisher Scientific, Inc., Waltham, MA, USA). cDNA was synthesized utilizing an RNeasy Mini Kit (Takara, Kyoto, Japan). qPCR analysis was executed with a SYBR Green Master Mix (Takara) with a program of $95^{\circ} \mathrm{C}$ for $5 \mathrm{~min}, 45$ cycles of $95^{\circ} \mathrm{C}$ for $5 \mathrm{sec}$, and $60^{\circ} \mathrm{C}$ for $30 \mathrm{sec} ; 1 \mathrm{cycle}$ of $95^{\circ} \mathrm{C}$ for $5 \mathrm{sec}$, $60^{\circ} \mathrm{C}$ for $1 \mathrm{~min}$, and $95^{\circ} \mathrm{C}$ for $15 \mathrm{sec}$; and, finally, $50^{\circ} \mathrm{C}$ for $30 \mathrm{sec}$. The $2-\Delta \Delta \mathrm{Cq}$ method was used to analyze relative expression. Expression of mRNAs was normalized to $\beta$-actin. The primers were designed with the online tool (https:// www.genscript.com/tools/real-time-pcr-tagman-primerdesign-tool) and synthesized by Shanghai Generay Biotech Co. Ltd. (Shanghai, China). Primers are listed in Table 1.

2.12. Validation and Survival Analysis of Key Hub Genes. To validate both the RNA expression level and the protein level of hub genes, the website server GEPIA (Gene Expression Profiling Interactive Analysis) [45] and the Human Protein Atlas database [46] were utilized. With the help of the GEPIA website, the relative RNA expression level between ccRCC tissue and normal renal tissue was visualized with box plots.
The Human Protein Atlas database was used to map the proteins in the tissues. Finally, the survival analysis of ccRCC patients was performed using TCGA data from the GEPIA website as well.

\section{Results}

3.1. Basic Characteristics of Microarray Data. The workflow of our study is shown in Figure 1. On the GPL570 ([HG-U133_Plus_2] Affymetrix Human Genome U133 Plus 2.0 Array) platform, we screened out two data sets (GSE53757 and GSE73731) with pathological stage information. Each data set had data from more than 50 patients. All raw data files were assessed with the RLE and NUSE algorithms. Finally, excluding the data files that did not satisfy the requirements, a total of 261 raw data files were filtered out. These data were divided into five groups: ccRCC stages I, II, III, and IV and normal tissues. The normal tissue group had 72 raw data files, and ccRCC stages I, II, III and IV had $62,29,41$, and 57 raw data files, respectively (Table 2).

3.2. Identification of DEGs. The gene expression levels of the ccRCC stage I, II, III, and IV tissues were compared with those of normal renal tissues separately. After background correction, normalization, and logarithmic conversion by conducting RMA, we obtained 1410 DEGs between normal 

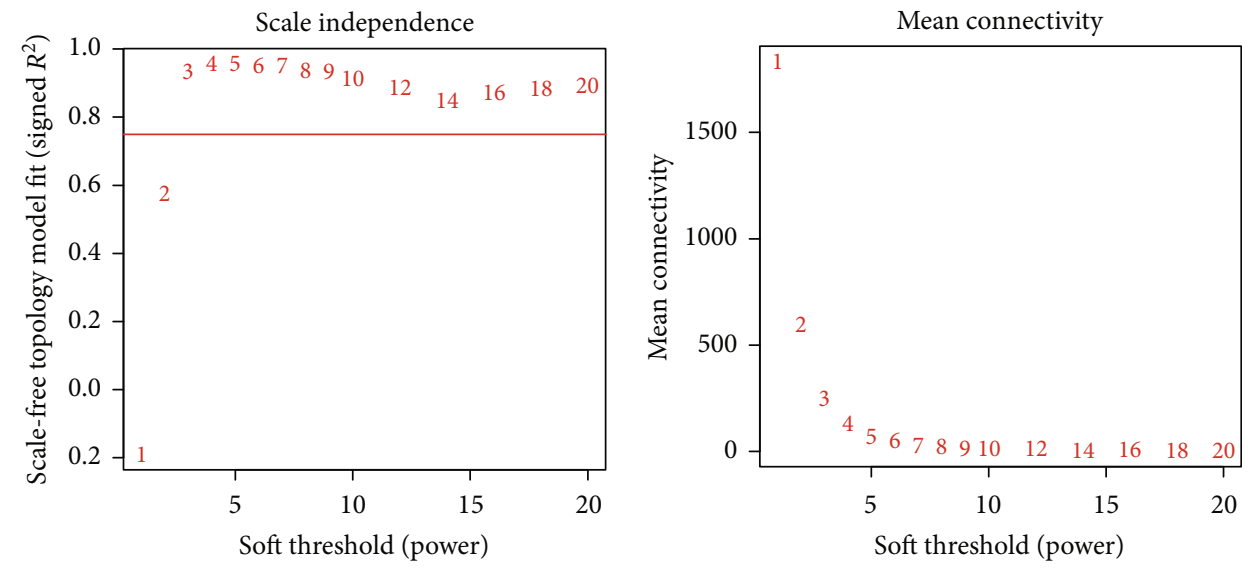

(a)

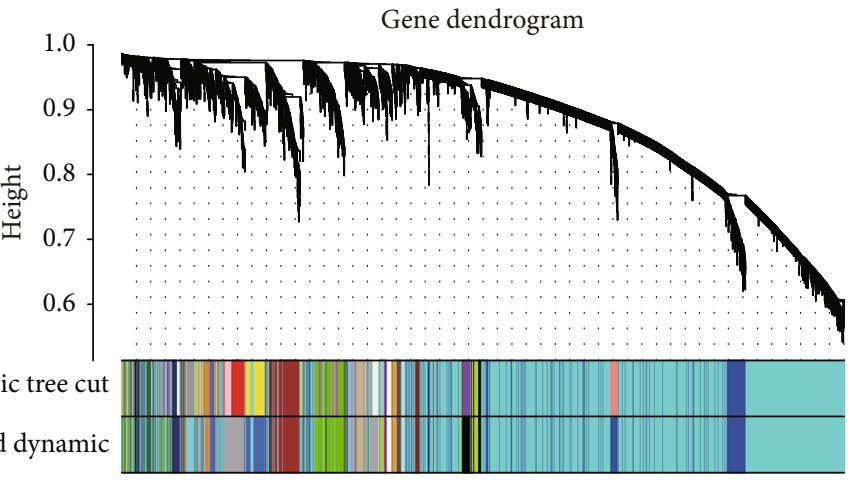

(b)
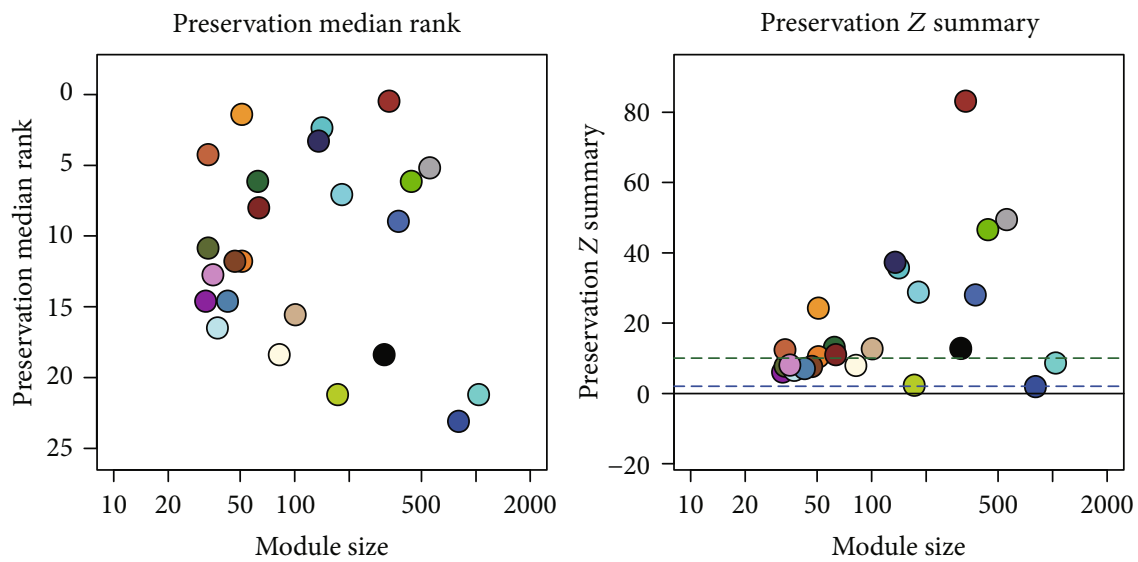

(c)

Figure 3: Continued. 


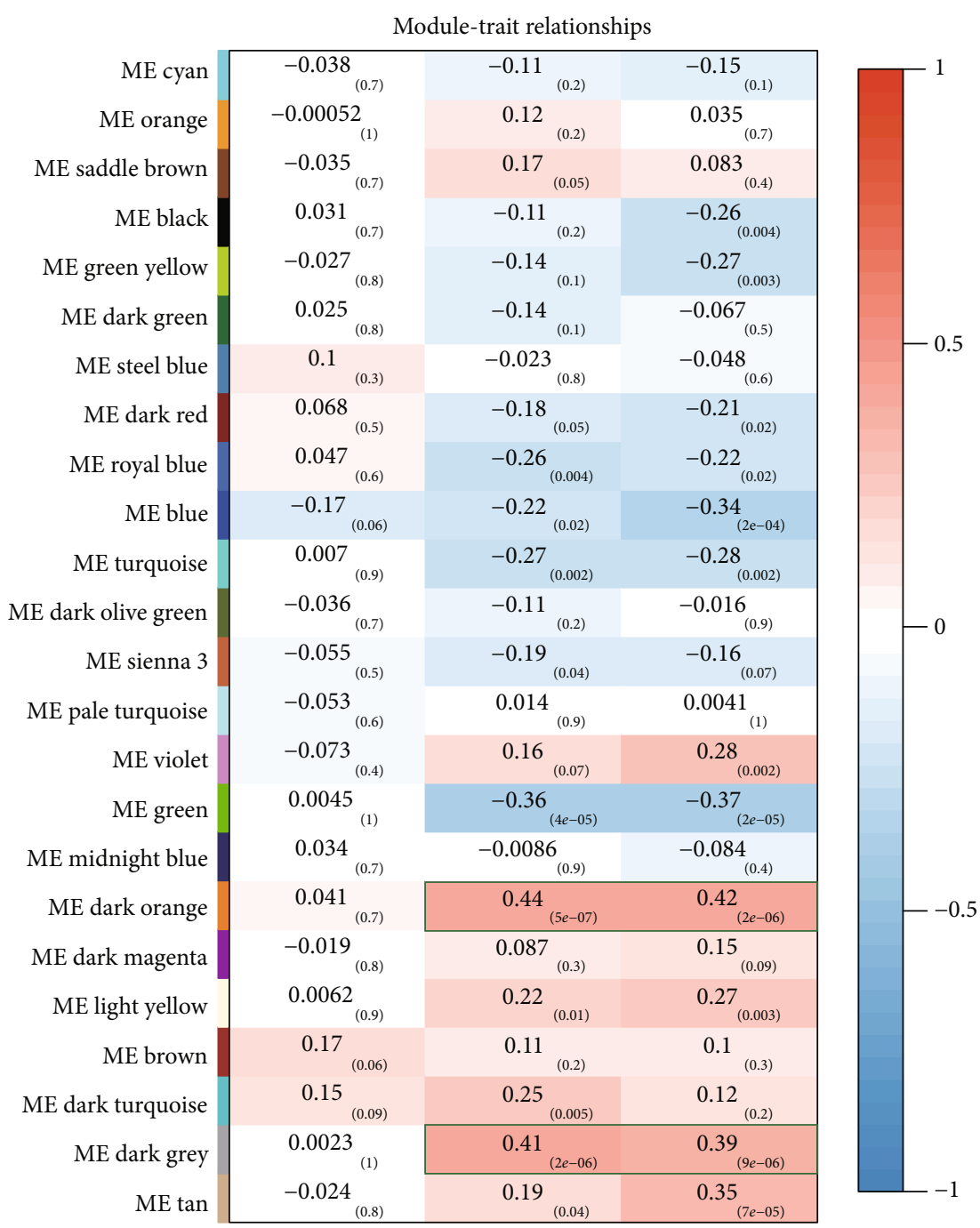

(d)

Gene significance across modules, $P$ value $=2.1 e-138$

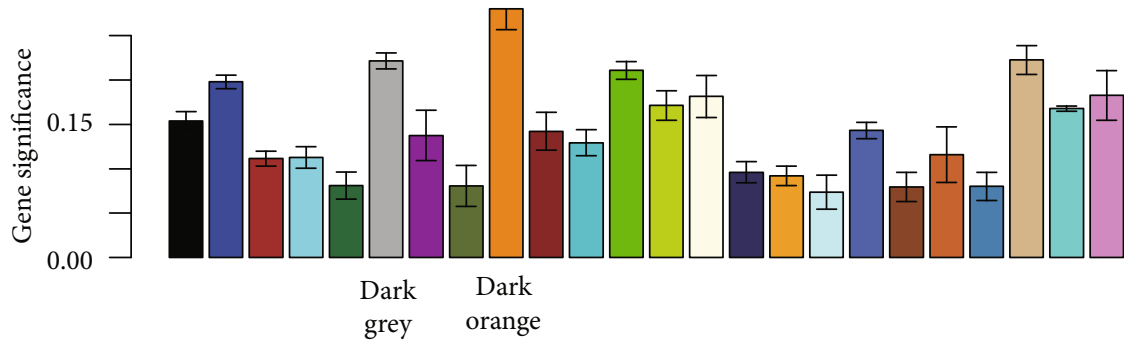

(e)

FIGURE 3: Weighted gene coexpression analysis for GSE73731. (a) Analysis of the scale-free fit index and mean connectivity for various softthresholding powers. (b) The clustering dendrogram based on the dissimilarity (1-TOM) using the dynamic tree cut method. (c) Module preservation test with an independent dataset, GSE53757. (d) Module-trait relationship heat map based on the Pearson correlation coefficient between module eigengenes and clinical parameters (age, grade, and stage). (e) Barplot for average gene significance across modules associated with the pathological stage for ccRCC.

tissue and ccRCC stage I, 632 DEGs between normal tissue and ccRCC stage II, 1915 DEGs between normal tissue and ccRCC stage III, and 1388 DEGs between normal tissue and ccRCC stage IV with the cut-off criteria of $|\log 2 \mathrm{FC}|$
$>2$ and an adjusted $P$ value $<0.05$. Then, as shown in Figure 2(a), 500 common DEGs were extracted from the 4 comparison groups. Among these 500 DEGs, 407 genes were upregulated and 93 genes were downregulated. 


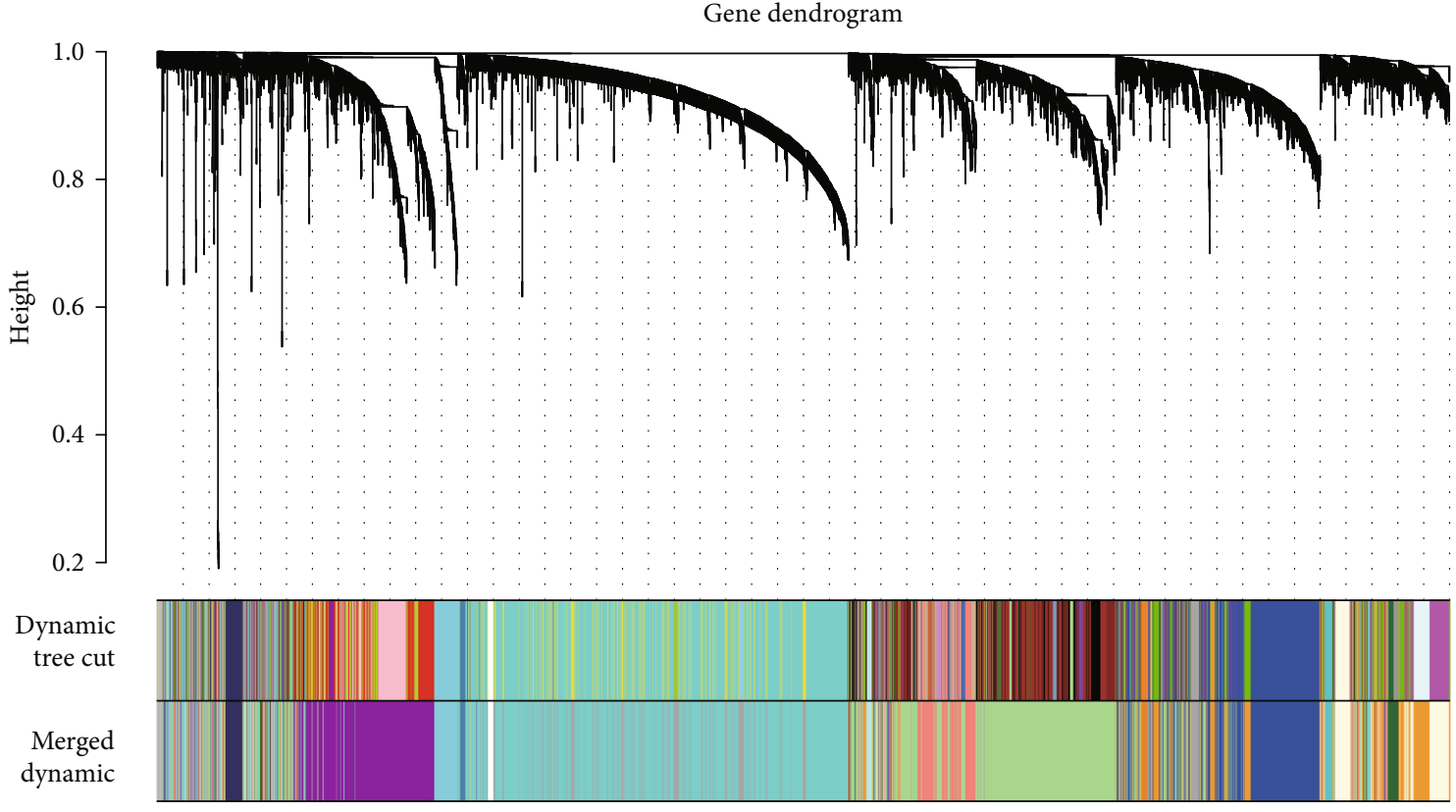

(a)

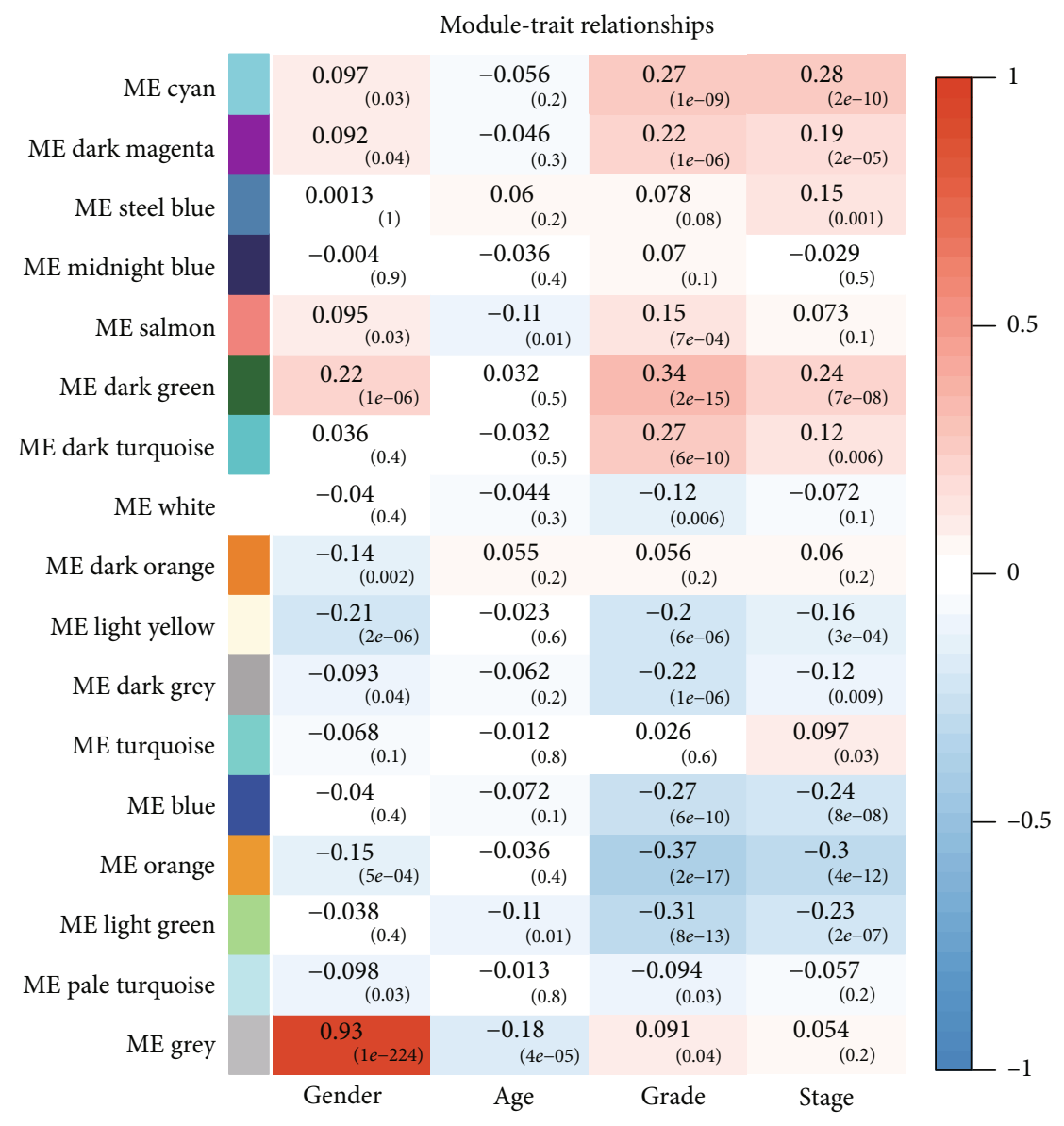

(b)

Figure 4: Continued. 
Gene significance across modules, $P$ value $=0$

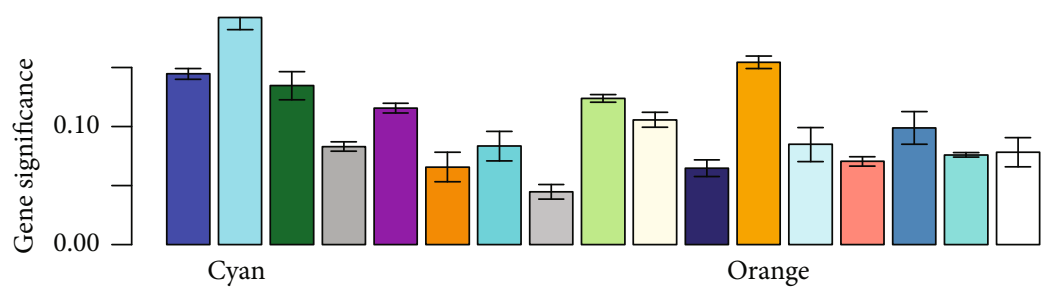

(c)

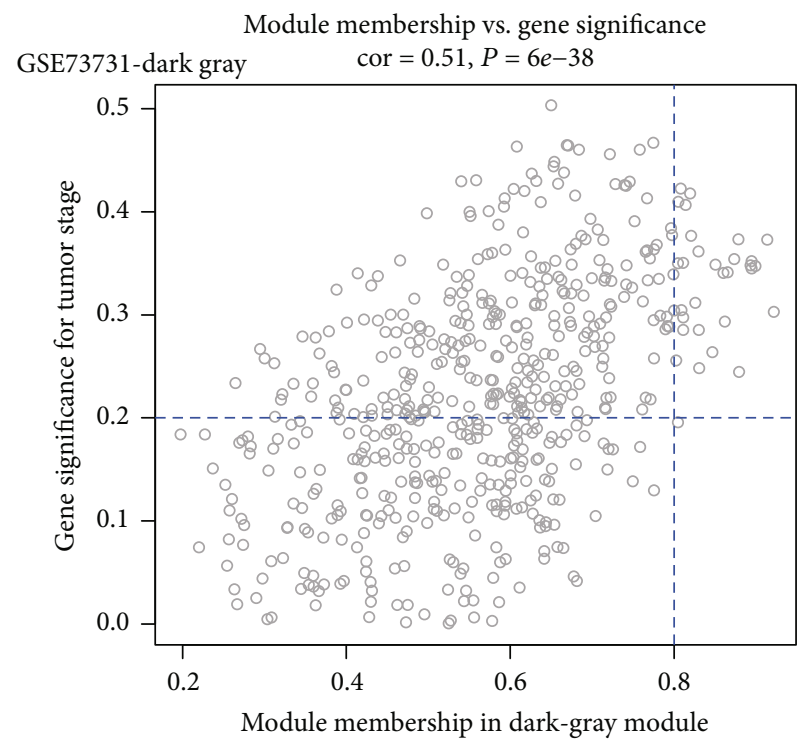

(d)

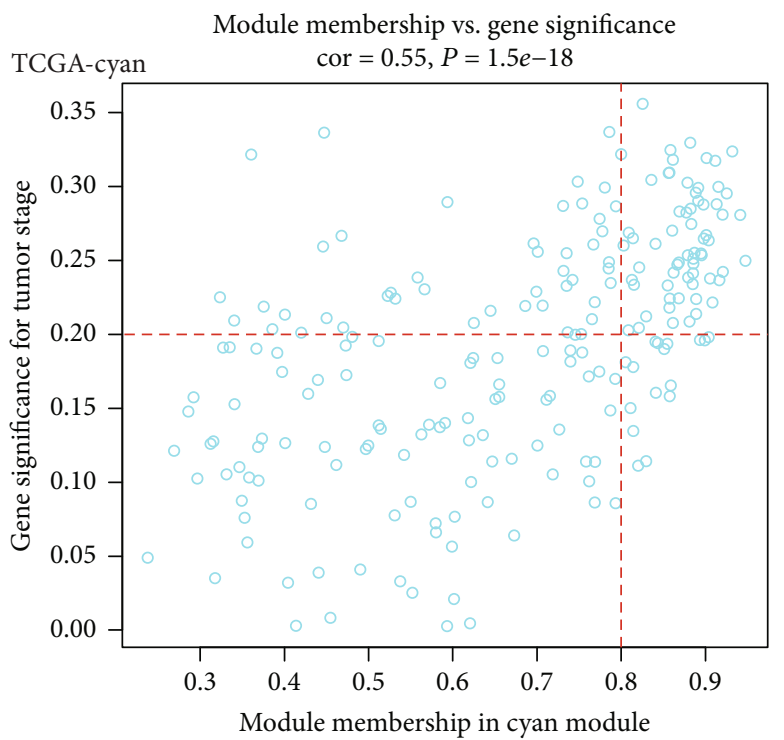

(e)

FIgURe 4: Weighted gene coexpression analysis for TCGA and screening for intramodular hub genes of key modules. (a) The clustering dendrogram based on the dissimilarity (1-TOM) using the dynamic tree cut method. (b) Module-trait relationship heat map based on the Pearson correlation coefficient between module eigengenes and clinical parameters (gender, age, grade, and stage). (c) Barplot for average gene significance across modules associated with the pathological stage for ccRCC. (d) Scatter plot of module eigengenes in the dark-gray module from GSE73731. (e) Scatter plot of module eigengenes in the cyan module from TCGA. Dots in the upper-right corner in both plots represent hub genes in the module.

3.3. Functional Enrichment Analyses of DEGs. With the clusterProfiler package in R software, we conducted GO and KEGG pathway enrichment analyses to shed new light on the functions of the identified DEGs. We uploaded the lists of DEGs, and the results of the GO analysis showed that these genes were enriched in different functional categories: BP, MF, and CC. The top 20 significant terms of each functional category are plotted in Figure 2(b). Most of the DEGs were enriched in "leukocyte migration," "receptor ligand activity," and "extracellular matrix" terms. Regarding the KEGG pathways, most of the DEGs were associated with the "PI3K-Akt signaling pathway" and "cytokine-cytokine receptor interaction," which was consistent with the biological process terms (Figure 2(c)).

3.4. Construction of PPI Networks. PPI networks of DEGs were built in accordance with the online database STRING and visualized in Cytoscape software with the minimum required interaction score of 0.700 . The PPI networks of 500 DEGs were composed of 250 nodes and 1214 edges after excluded lowest clustering score nodes (Figure 2(d)). Pink nodes indicated upregulated DEGs, while blue nodes indicated downregulated genes.

3.5. Weighted Gene Coexpression Network Analysis Using GSE73731. The WGCNA was performed to analyze the GSE73731 microarray data and the TCGA RNA-seq data. Both datasets were properly normalized and filtered to reduce outliers.

After data preprocessing, 121 samples with complete clinical information and 9420 varying genes were analyzed in the GSE73731 dataset by WGCNA. Constructing a weighted gene network entailed the choice of the softthresholding power to which coexpression similarity was raised to calculate adjacency. Using the pickSoftThreshold function in the WGCNA package, we selected 3 as the best $\beta$ for the following analysis (Figure 3(a)). As we assigned all of the genes to their corresponding modules, 23 modules were detected in the GSE73731 dataset (Figure 3(b)). According to the module-trait heat map (Figure 3(d)), we discovered 
TABLE 3: Four key hub genes selected from among 500 DEGs and 21 common hub genes from GSE73731 and TCGA-KIRC data.

\begin{tabular}{|c|c|c|c|c|c|}
\hline Gene symbol & Description & $\begin{array}{c}\text { Log FC in } \\
\text { stage I }\end{array}$ & $\begin{array}{c}\text { Log FC in } \\
\text { stage II }\end{array}$ & $\begin{array}{c}\text { Log FC in } \\
\text { stage III }\end{array}$ & $\begin{array}{c}\text { Log FC in } \\
\text { stage IV }\end{array}$ \\
\hline UBE2C & Ubiquitin-conjugating enzyme E2C & 2.92 & 2.60 & 3.68 & 3.76 \\
\hline BUB1B & BUB1 mitotic checkpoint serine/threonine kinase B & 3.07 & 2.24 & 3.26 & 3.19 \\
\hline RRM2 & Ribonucleotide reductase regulatory subunit M2 & 3.08 & 2.9 & 3.74 & 4.10 \\
\hline TPX2 & TPX2 microtubule nucleation factor & 2.59 & 2.51 & 3.14 & 3.50 \\
\hline
\end{tabular}

TABLE 4: Histopathological characteristics of the ccRCC patients.

\begin{tabular}{lccccc}
\hline Patient & Sex & Age (years) & Pathology & Location & Tumor size $(\mathrm{cm})$ \\
\hline 1 & Male & 65 & ccRCC, partially PRCC & Left & $12 * 10.5$ \\
2 & Male & 80 & ccRCC & Left & $14.8 * 9.8$ \\
3 & Female & 58 & ccRCC, partially PRCC & Left & $7 * 4 * 2.5$ \\
4 & Male & 63 & ccRCC & Right & $4.5 * 4.5$ \\
5 & Female & 81 & ccRCC, partially PRCC & Left & IV \\
6 & Female & 56 & ccRCC & Right & $2.5 * 3.1$ \\
7 & Male & 57 & ccRCC & Right & IV \\
8 & Male & 34 & ccRCC & Right & IV \\
\hline
\end{tabular}

Note: ccRCC: clear cell renal cell carcinoma; PRCC: papillary renal cell carcinoma.

that the dark-orange and dark-gray modules were most related to tumor stage as well as tumor grade. In addition, Figure 3(e) showed that the gene significance with the tumor stage of these two modules was also the highest across modules. The sequence preservation test indicated that the dark-gray module was well preserved in the GSE53757 dataset; however, the dark-orange module was preserved (Figure 3(c)). Considering that the dark-orange module had a relatively small size and poor preservation, we chose the dark-gray module as our target module that contained 553 genes in this module.

3.6. Weighted Gene Coexpression Network Analysis Using TCGA-KIRC Dataset and Filtration of Hub Genes. Since the GSE73731 dataset had relatively few data with a clinical stage phenotype, we used the TCGA-KIRC dataset to verify the hub genes by constructing another coexpression network. With the TCGA-KIRC dataset, the network was composed of 12,000 gene expression profiles derived from 502 KIRC tumor tissues. Following the above procedure, we clustered genes into 16 different modules (Figure 4(a)) and determined that the cyan module was most related to the tumor stage phenotype (Figures 4(b) and 4(c)). Using the criteria mentioned earlier, we filtered out 29 hub genes (Figure 4(d)) in the dark-gray module with high module membership and gene significance. With the same criteria, 63 genes in the cyan module were identified as hub genes (Figure 4(e)). When we combined the hub genes from the GSE73731 and TCGAKIRC data, 21 genes were found in common. Finally, we took the intersection of the common hub genes and DEGs from the previous section. Four genes that were identified as DEGs were also presented among the common hub genes (Table 3).
3.7. Validation of RNA and Protein Expression Level for Key Hub Genes. Table 4 showed the histopathological characteristics of ccRCC patients. Experimental validation of UBE2C, BUB1B, RRM2, and TPX2 was successful in 8 paired clinical ccRCC samples, demonstrating that all of them were upregulated in ccRCC (Figure 5(a)). As for the protein expression level's validation, it was assessed to indicate that UBE2C and TPX2 expressions were significantly higher in tumor tissues than in normal tissues in accordance with the Human Protein Atlas database (Figure 5(b) and Table 5). Besides, it could be seen from the microscope's photographs of IHC staining that there was a clear boundary between the cells of normal kidney tissues, while the cell morphology of kidney cancer tissues exhibited diversities, and nuclear heteromorphs could be observed as well.

3.8. Validation and Survival Analysis of Key Hub Genes. The RNA expression levels of UBE2C, BUB1B, RRM2, and TPX2 were also validated in the TCGA dataset. With the cut-off criteria of $|\log 2 \mathrm{FC}|>1$ and $P<0.01, \mathrm{UBE} 2 \mathrm{C}$, BUB1B, RRM2, and TPX2 were upregulated in $523 \mathrm{ccRCC}$ samples as compared to 72 normal samples (Figure 6(a)). Meanwhile, pathological stage plots showed that UBE2C, BUB1B, RRM2, and TPX2 were significantly correlated with the stage of ccRCC (Figure 6(b)). These genes were more abundantly expressed in advanced kidney cancer as compared to early ccRCC, which was consistent with the above analysis. In Figure 6(c), we analyzed the overall survival (OS) of UBE2C, BUB1B, RRM2, and TPX2 in ccRCC. The lower expression levels of hub genes predicted better prognosis of ccRCC than higher expression. 

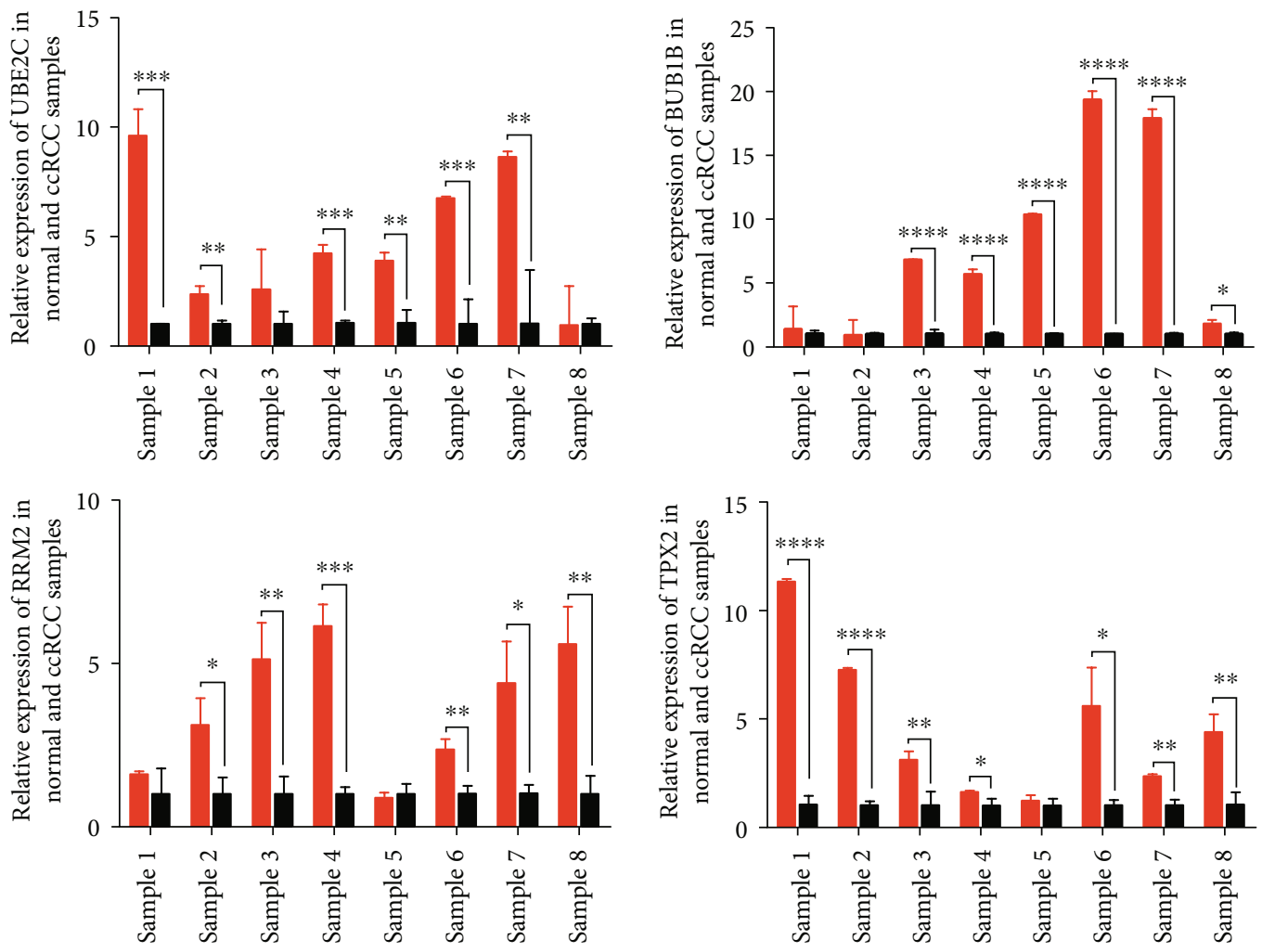

Tumor

(a)
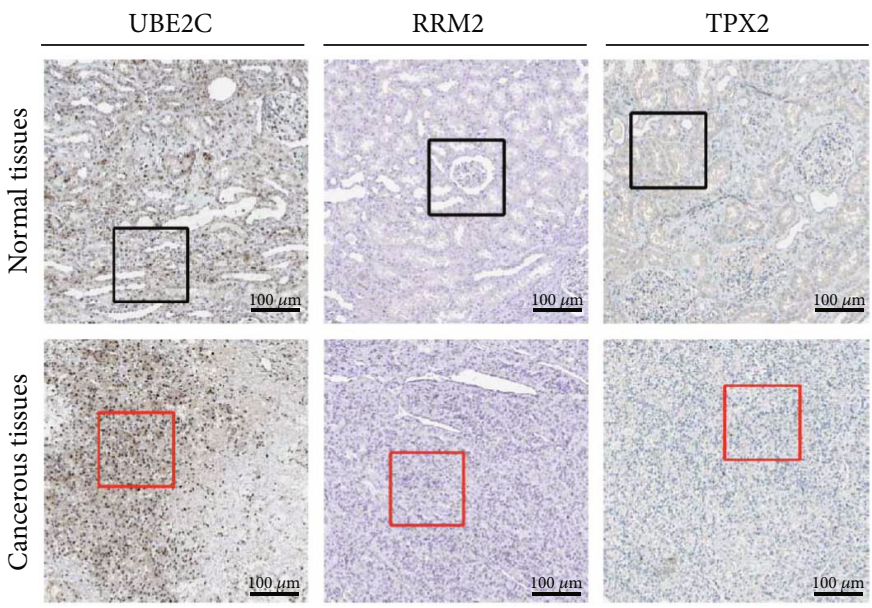

(b)

Figure 5: Validation of RNA and protein expression levels for hub genes. (a) Experimental validation of UBE2C, BUB1B, RRM2, and TPX2 in 8 paired clinical ccRCC samples. Data are presented as the means \pm SEM. ${ }^{*} P<0.05,{ }^{* *} P<0.01,{ }^{* * *} P<0.001$, and ${ }^{* * * *} P<0.0001$. (b) UBE2C, RRM2, and TPX2 protein expression levels in ccRCC as compared to those in normal kidney tissues by IHC staining. Scale bars represent $100 \mu \mathrm{m}$. Black and red square boxes represent typical normal kidney and ccRCC tissues, respectively.

\section{Discussion}

To explore the occurrence and development of diseases from a novel perspective, bioinformatics analysis is becoming an emerging and effective technique $[47,48]$. Clear cell renal cell carcinoma is a major histological subtype of renal cell carci- noma, which is one of the deadliest forms of urinary malignancy [1]. Sequential changes in gene expression in different clinical stages play essential roles in ccRCC. In recent decades, strides have been made in identifying pathogenesis-related genes and therapeutic potential biomarkers of ccRCC $[26,49,50]$. However, most of them only 
TABle 5: IHC staining characteristics of hub genes from the Human Protein Atlas database.

\begin{tabular}{|c|c|c|c|c|c|c|c|}
\hline Gene & Patient ID & Gender & Age (years) & Staining & Intensity & Quantity & Location \\
\hline \multicolumn{8}{|c|}{ Normal tissues } \\
\hline UBE2C & 3229 & Male & 59 & Medium & Moderate & $>75 \%$ & $\begin{array}{c}\text { Cytoplasmic } \\
\text { Membranous } \\
\text { Nuclear }\end{array}$ \\
\hline RRM2 & 1859 & Male & 61 & Not detected & Negative & None & None \\
\hline TPX2 & 1933 & Female & 56 & Low & Weak & $25-75 \%$ & $\begin{array}{l}\text { Cytoplasmic } \\
\text { Membranous }\end{array}$ \\
\hline \multicolumn{8}{|c|}{ Cancerous tissues } \\
\hline UBE2C & 2540 & Male & 61 & High & Strong & $>75 \%$ & $\begin{array}{c}\text { Cytoplasmic } \\
\text { Membranous } \\
\text { Nuclear }\end{array}$ \\
\hline RRM2 & 3616 & Female & 63 & Not detected & Negative & None & None \\
\hline TPX2 & 1831 & Male & 77 & Medium & Strong & $25 \%$ & Nuclear \\
\hline
\end{tabular}

Note: IHC: immunohistochemistry.

look into how genes were dysregulated between cancerous and paracancerous tissues. Our study has a more detailed grouping, as there are five comparison groups in total. The gene expression levels in ccRCC stages I, II, III, and IV are compared with those of normal renal tissues. More precisely, we filter four genes highly related to clinical stages by WGCNA and multidimensional validations.

In our study, we downloaded the gene expression profiles of GSE53757 and GSE73731 and screened 500 DEGs involved in the development of ccRCC. GO terms of BP revealed that most of the DEGs were enriched in inflammation- and immunity-related categories. KEGG pathway analysis revealed that the DEGs were mainly involved in the "PI3K-Akt signaling pathway," "cytokine-cytokine receptor interaction," and "focal adhesion" pathways. The above results further suggest that ccRCC is a disease associated with cytokinesis and inflammation [51, 52], providing bioinformatics evidence for deep research. Moreover, aiming to make our results more accurate, WGCNA was performed in GSE73731 to identify gene coexpression modules related to clinical stages of ccRCC, and the modules were preserved in GSE53757. The dark-gray module was confirmed, and hub genes were derived from this module. By the same token, TCGA-KIRC data were also downloaded to perform WGCNA, and the corresponding results were obtained.

After that, we obtained 4 key hub genes of interest that overlapped between 500 DEGs and 21 common hub genes selected in the GSE73731 and TCGA-KIRC data by WGCNA, including UBE2C, BUB1B, RRM2, and TPX2. Although previous studies have reported the association of these genes with ccRCC, most of them either used simple methods or lacked experimental verification, reducing the credibility of their results. For instance, overexpressed RRM2 was experimentally confirmed to be associated with a trend toward the advanced pathological stage, high Fuhrman grade, and poor prognosis [53]. In addition, a recent study reported that miR-99a-3p could regulate RRM2 in sunitinib-resistant ccRCC, showing a potential antitumor effect [54]. Analyzing TCGA and tissue microarray data showed that TPX2 was associated with advanced grade and stage of ccRCC, which could be a potential therapeutic target as well [55]. These results are consistent with ours. As for UBE2C and BUB1B, Yuan et al. reported that UBE2C and five other genes identified in WGCNA and PPI networks were highly related to progression and poor prognosis of ccRCC [56]. However, they did not validate the genes in any experiment. Studies on BUB1B in ccRCC are rare. Although a study reported that along with 31 other genes, the four genes that we screened out were related to metastasis of ccRCC [57], it still had no validation. In our study, we screened UBE2C, BUB1B, RRM2, and TPX2 as key hub genes of ccRCC by integrated bioinformatics analysis. Experimental validation of RNA and protein levels showed that they were higher in cancerous than in paracancerous tissues. Overexpression of the four genes was also associated with an advanced clinical stage and poor overall survival rate. Thus, the neoteric key hub genes discovered in our study are likely to become a group of potential therapeutic targets of ccRCC.

Compared with other studies of ccRCC, our study is innovative in several aspects. Integrated bioinformatics analyses were applied to shed light on the relationship between DEGs and tumor clinical features in ccRCC. Moreover, we combined two gene expression microarray datasets from the GEO database and one RNA-seq dataset with corresponding clinical information from TCGA to screen out tumor stage-relevant DEGs of ccRCC. Moreover, the intersection of hub genes selected from key modules of WGCNA and DEGs selected from GEO datasets helped us identify 4 highly believable and accurate genes, which further validated the relevance between the genes and the progression and prognosis in ccRCC by multiple methods. However, a limitation of our approach is that further biological experiments on such aspects need to be carried out to verify the function of these potential biomarkers.

\section{Conclusions}

In conclusion, the present study identified key hub genes associated with the clinical stage and overall survival of ccRCC patients. Our study offers a deeper understanding of 

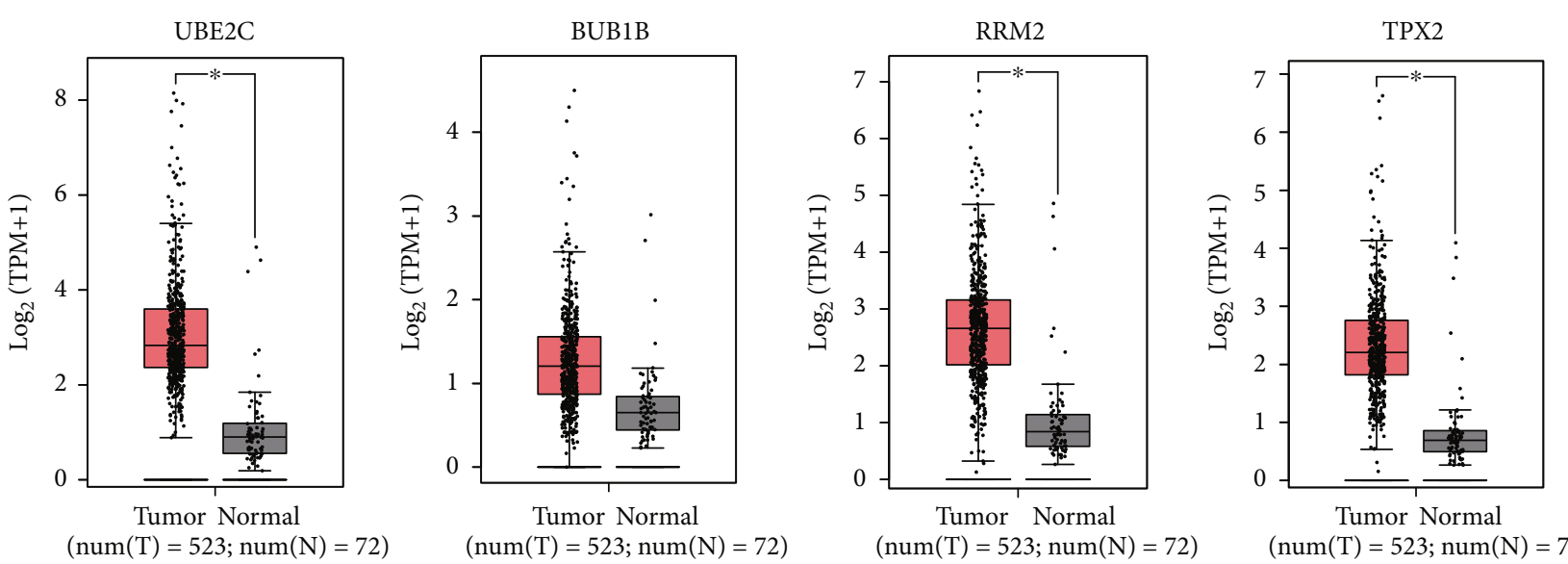

(a)
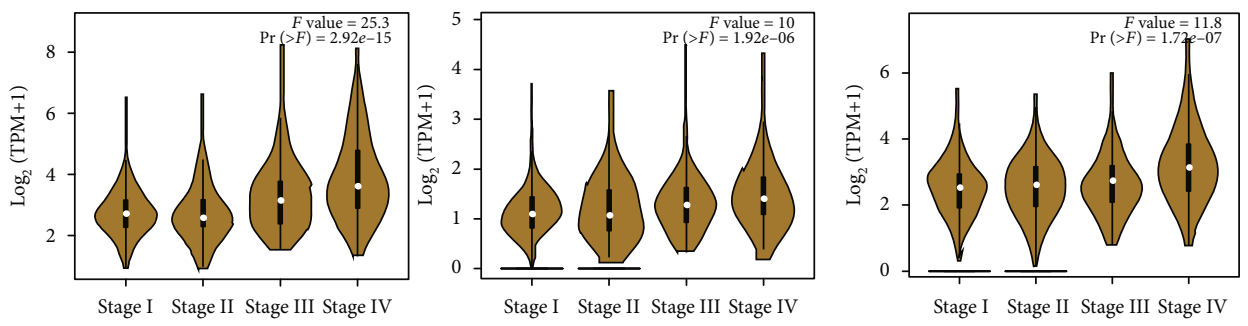

(b)
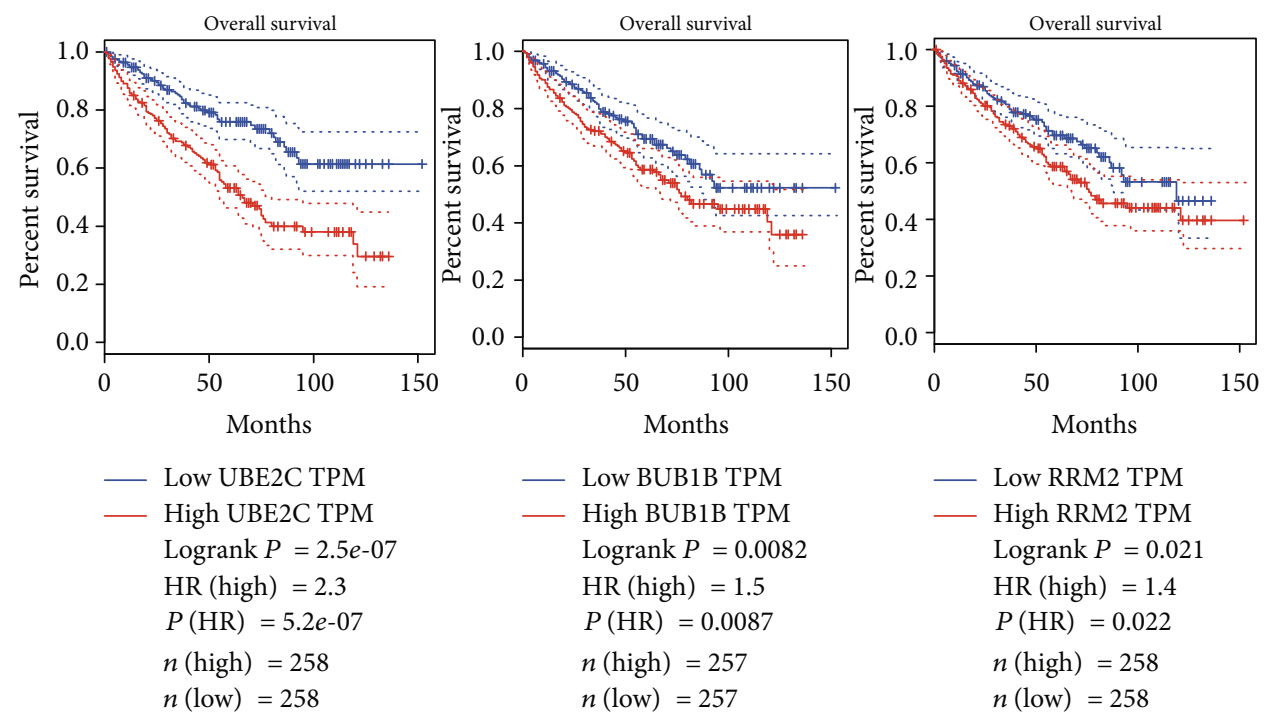
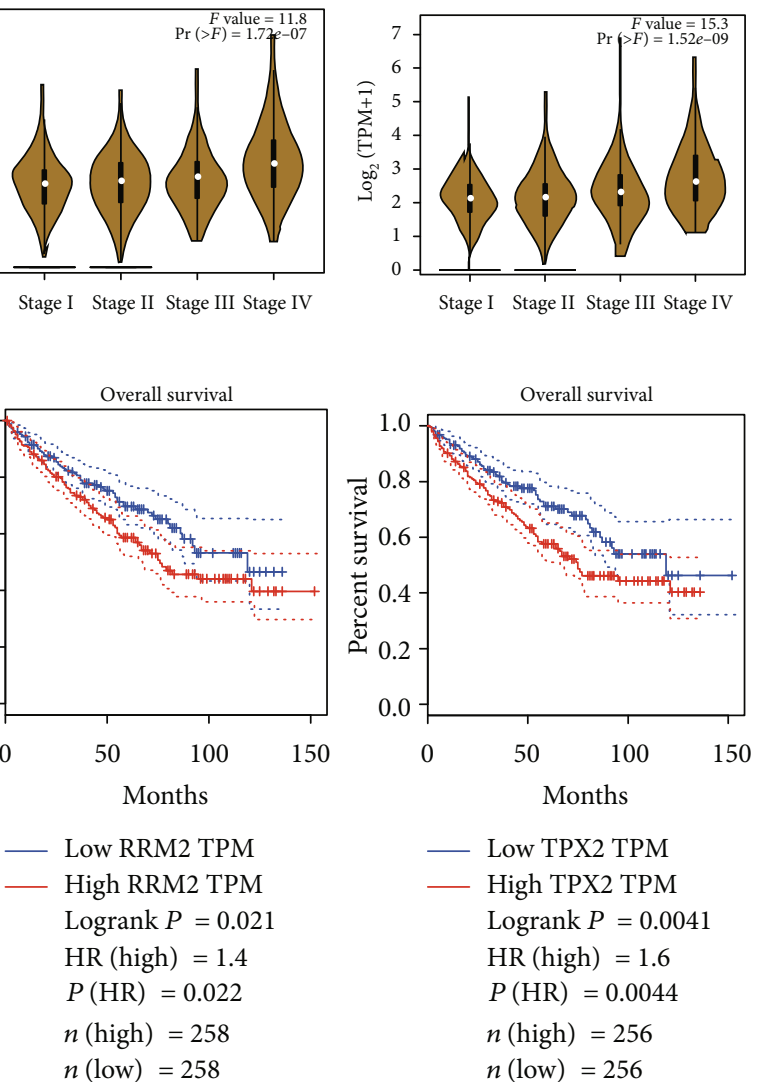

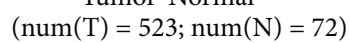


F.W., and Y.L.L. contributed to interpretation of the results and prepared the figures. M.Z. contributed to drafting the manuscript. All authors reviewed and edited the manuscript. All authors listed in the manuscript have concurred with the content of the manuscript and submission. D.X. and Y.X. contributed equally to this work.

\section{Acknowledgments}

We thank the institutions managing the TCGA, GEO, and other databases for the freely accessible datasets without limitations. This work was supported by the Zhejiang Medical and Health Science and Technology Project (2015DTA017) and Zhejiang Science and Technology Plan Project (2017C37106).

\section{References}

[1] R. L. Siegel, K. D. Miller, and A. Jemal, "Cancer statistics, 2018," CA: a Cancer Journal for Clinicians, vol. 68, no. 1, pp. 7-30, 2018.

[2] U. Capitanio and F. Montorsi, "Renal cancer," Lancet, vol. 387, no. 10021, pp. 894-906, 2016.

[3] J. R. Srigley, B. Delahunt, J. N. Eble et al., "The international society of urological pathology (ISUP) vancouver classification of renal neoplasia," The American Journal of Surgical Pathology, vol. 37, no. 10, pp. 1469-1489, 2013.

[4] R. J. Motzer, E. Jonasch, N. Agarwal et al., "Kidney cancer, version 3.2015," Journal of the National Comprehensive Cancer Network, vol. 13, no. 2, pp. 151-159, 2015.

[5] H. T. Cohen and F. J. McGovern, "Renal-cell carcinoma," The New England Journal of Medicine, vol. 353, no. 23, pp. 24772490, 2005.

[6] C. Suarez, R. Morales, E. Munoz, J. Rodon, C. M. Valverde, and J. Carles, "Molecular basis for the treatment of renal cell carcinoma," Clinical \& Translational Oncology, vol. 12, no. 1, pp. 15-21, 2010.

[7] H. Hase, K. Jingushi, Y. Ueda et al., "LOXL2 status correlates with tumor stage and regulates integrin levels to promote tumor progression in ccRCC," Molecular Cancer Research, vol. 12, no. 12, pp. 1807-1817, 2014.

[8] V. A. Valera and M. J. Merino, "Misdiagnosis of clear cell renal cell carcinoma," Nature Reviews Urology, vol. 8, no. 6, pp. 321333, 2011.

[9] B. Escudier, T. Eisen, W. M. Stadler et al., "Sorafenib for treatment of renal cell carcinoma: final efficacy and safety results of the phase III treatment approaches in renal cancer global evaluation trial," Journal of Clinical Oncology, vol. 27, no. 20, pp. 3312-3318, 2009.

[10] K. T. Flaherty, J. B. Manola, M. Pins et al., "BEST: a randomized phase II study of vascular endothelial growth factor, RAF kinase, and mammalian target of rapamycin combination targeted therapy with bevacizumab, sorafenib, and temsirolimus in advanced renal cell carcinoma-a trial of the ECOGACRIN Cancer Research Group (E2804)," Journal of Clinical Oncology, vol. 33, no. 21, pp. 2384-2391, 2015.

[11] D. Y. Heng, W. Xie, M. M. Regan et al., "External validation and comparison with other models of the International Metastatic Renal-Cell Carcinoma Database Consortium prognostic model: a population-based study," The Lancet Oncology, vol. 14, no. 2, pp. 141-148, 2013.
[12] B. Shuch, A. Amin, A. J. Armstrong et al., "Understanding pathologic variants of renal cell carcinoma: distilling therapeutic opportunities from biologic complexity," European Urology, vol. 67, no. 1, pp. 85-97, 2015.

[13] R. P. Theis, S. M. Dolwick Grieb, D. Burr, T. Siddiqui, and N. R. Asal, "Smoking, environmental tobacco smoke, and risk of renal cell cancer: a population-based case-control study," BMC Cancer, vol. 8, no. 1, 2008.

[14] I. A. Deckers, P. A. van den Brandt, M. van Engeland et al., "Polymorphisms in genes of the renin-angiotensinaldosterone system and renal cell cancer risk: interplay with hypertension and intakes of sodium, potassium and fluid," International Journal of Cancer, vol. 136, no. 5, pp. 11041116, 2015.

[15] A. Gati, S. Kouidhi, R. Marrakchi et al., "Obesity and renal cancer: role of adipokines in the tumor-immune system conflict," Oncoimmunology, vol. 3, no. 1, article e27810, 2014.

[16] M. M. Baldewijns, I. J. van Vlodrop, L. J. Schouten, P. M. Soetekouw, A. P. de Bruine, and M. van Engeland, "Genetics and epigenetics of renal cell cancer," Biochimica et Biophysica Acta, vol. 1785, no. 2, pp. 133-155, 2008.

[17] J. R. Gnarra, K. Tory, Y. Weng et al., "Mutations of the VHL tumour suppressor gene in renal carcinoma," Nature Genetics, vol. 7, no. 1, pp. 85-90, 1994.

[18] I. Varela, P. Tarpey, K. Raine et al., "Exome sequencing identifies frequent mutation of the SWI/SNF complex gene PBRM1 in renal carcinoma," Nature, vol. 469, no. 7331, pp. 539-542, 2011.

[19] A. Högner, H. Krause, B. Jandrig et al., "PBRM1 and VHL expression correlate in human clear cell renal cell carcinoma with differential association with patient's overall survival," Urologic Oncology: Seminars and Original Investigations, vol. 36, no. 3, pp. 94.e1-94.e14, 2018.

[20] G. Guo, Y. Gui, S. Gao et al., "Frequent mutations of genes encoding ubiquitin-mediated proteolysis pathway components in clear cell renal cell carcinoma," Nature Genetics, vol. 44, no. 1, pp. 17-19, 2011.

[21] S. Peña-Llopis, S. Vega-Rubín-de-Celis, A. Liao et al., "BAP1 loss defines a new class of renal cell carcinoma," Nature Genetics, vol. 44, no. 7, pp. 751-759, 2012.

[22] Y. C. Wi, A. Moon, M. J. Jung et al., "Loss of nuclear BAP1 expression is associated with high WHO/ISUP grade in clear cell renal cell carcinoma," Journal of Pathology and Translational Medicine, vol. 52, no. 6, pp. 378-385, 2018.

[23] W. H. da Costa, A. F. Fares, S. M. Bezerra et al., "Loss of BAP1 expression in metastatic tumor tissue is an event of poor prognosis in patients with metastatic clear cell renal cell carcinoma," Urologic Oncology: Seminars and Original Investigations, vol. 37, no. 1, pp. 78-85, 2019.

[24] P. Langfelder and S. Horvath, "WGCNA: an R package for weighted correlation network analysis," BMC Bioinformatics, vol. 9, no. 1, 2008 .

[25] Z. Yu, L. Ni, D. Chen et al., "Expression and clinical significance of RCDG1 in renal cell carcinoma: a novel renal cancer-associated gene," Molecular Medicine Reports, vol. 10, no. 3, pp. 1583-1589, 2014.

[26] L. Yuan, G. Zeng, L. Chen et al., "Identification of key genes and pathways in human clear cell renal cell carcinoma (ccRCC) by co-expression analysis," International Journal of Biological Sciences, vol. 14, no. 3, pp. 266-279, 2018. 
[27] A. O. Osunkoya, Q. Yin-Goen, J. H. Phan et al., "Diagnostic biomarkers for renal cell carcinoma: selection using novel bioinformatics systems for microarray data analysis," Human Pathology, vol. 40, no. 12, pp. 1671-1678, 2009.

[28] M. H. Bui, D. Seligson, K. R. Han et al., "Carbonic anhydrase IX is an independent predictor of survival in advanced renal clear cell carcinoma: implications for prognosis and therapy," Clinical Cancer Research, vol. 9, no. 2, pp. 802-811, 2003.

[29] J. J. Patard, P. Fergelot, P. I. Karakiewicz et al., "Low CAIX expression and absence of VHL gene mutation are associated with tumor aggressiveness and poor survival of clear cell renal cell carcinoma," International Journal of Cancer, vol. 123, no. 2, pp. 395-400, 2008.

[30] L. Gautier, L. Cope, B. M. Bolstad, and R. A. Irizarry, "affy-analysis of Affymetrix GeneChip data at the probe level," Bioinformatics, vol. 20, no. 3, pp. 307-315, 2004.

[31] B. Bolstad, "Preprocessing and normalization for Affymetrix GeneChip expression microarrays," Methods in Microarray Normalization, pp. 41-59, 2008.

[32] R. A. Irizarry, B. Hobbs, F. Collin et al., "Exploration, normalization, and summaries of high density oligonucleotide array probe level data," Biostatistics, vol. 4, no. 2, pp. 249-264, 2003.

[33] M. E. Ritchie, B. Phipson, D. Wu et al., "limma powers differential expression analyses for RNA-sequencing and microarray studies," Nucleic Acids Research, vol. 43, no. 7, article e47, 2015.

[34] Y. Benjamini and Y. Hochberg, "Controlling the false discovery rate: a practical and powerful approach to multiple testing," Journal of the Royal statistical society: series $B$ (Methodological), vol. 57, no. 1, pp. 289-300, 1995.

[35] G. Yu, L. G. Wang, Y. Han, and Q. Y. He, “clusterProfiler: an R package for comparing biological themes among gene clusters," OMICS, vol. 16, no. 5, pp. 284-287, 2012.

[36] D. Szklarczyk, A. L. Gable, D. Lyon et al., "STRING v11: protein-protein association networks with increased coverage, supporting functional discovery in genome-wide experimental datasets," Nucleic Acids Research, vol. 47, no. D1, pp. D607D613, 2019.

[37] S. Killcoyne, G. W. Carter, J. Smith, and J. Boyle, "Cytoscape: a community-based framework for network modeling," Methods in Molecular Biology, vol. 563, pp. 219-239, 2009.

[38] C. W. Law, Y. Chen, W. Shi, and G. K. Smyth, "voom: precision weights unlock linear model analysis tools for RNA-seq read counts," Genome Biology, vol. 15, no. 2, p. R29, 2014.

[39] G. K. Smyth, "Limma: linear models for microarray data," in Bioinformatics and Computational Biology Solutions Using $R$ and Bioconductor, pp. 397-420, Springer, 2005.

[40] P. Langfelder and S. Horvath, "FastRFunctions for robust correlations and hierarchical clustering," Journal of Statistical Software, vol. 46, no. 11, 2012.

[41] B. Zhang and S. Horvath, "A general framework for weighted gene co-expression network analysis," Statistical Applications in Genetics and Molecular Biology, vol. 4, no. 1, 2005.

[42] A. M. Yip and S. Horvath, "Gene network interconnectedness and the generalized topological overlap measure," BMC Bioinformatics, vol. 8, no. 1, 2007.

[43] P. Langfelder, B. Zhang, and S. Horvath, "Defining clusters from a hierarchical cluster tree: the dynamic tree cut package for R," Bioinformatics, vol. 24, no. 5, pp. 719-720, 2008.
[44] P. Langfelder, R. Luo, M. C. Oldham, and S. Horvath, "Is my network module preserved and reproducible?," PLoS Computational Biology, vol. 7, no. 1, article e1001057, 2011.

[45] Z. Tang, C. Li, B. Kang, G. Gao, C. Li, and Z. Zhang, "GEPIA: a web server for cancer and normal gene expression profiling and interactive analyses," Nucleic Acids Research, vol. 45, no. W1, pp. W98-W102, 2017.

[46] P. J. Thul and C. Lindskog, "The human protein atlas: a spatial map of the human proteome," Protein Science, vol. 27, no. 1, pp. 233-244, 2018.

[47] X. Yang, M. Jia, Z. Li et al., "Bioinformatics analysis of aggressive behavior of breast cancer via an integrated gene regulatory network," Journal of Cancer Research and Therapeutics, vol. 10, no. 4, pp. 1013-1018, 2014.

[48] J. Martinez-Romero, S. Bueno-Fortes, M. Martín-Merino, A. Ramirez de Molina, and J. de Las Rivas, "Survival marker genes of colorectal cancer derived from consistent transcriptomic profiling," BMC Genomics, vol. 19, Suppl 8, p. 857, 2018.

[49] Y. Xiong, L. Yuan, L. Chen et al., "Identifying a novel biomarker TOP2A of clear cell renal cell carcinoma (ccRCC) associated with smoking by co-expression network analysis," Journal of Cancer, vol. 9, no. 21, pp. 3912-3922, 2018.

[50] A. S. Parker, J. E. Eckel-Passow, D. Serie et al., "Higher expression of topoisomerase II alpha is an independent marker of increased risk of cancer-specific death in patients with clear cell renal cell carcinoma," European Urology, vol. 66, no. 5, pp. 929-935, 2014.

[51] Q. Lv, G. Wang, Y. Zhang et al., "FABP5 regulates the proliferation of clear cell renal cell carcinoma cells via the PI3K/AKT signaling pathway," International Journal of Oncology, vol. 54, no. 4, pp. 1221-1232, 2019.

[52] Z. Chen, K. Wang, H. Lu et al., "Systemic inflammation response index predicts prognosis in patients with clear cell renal cell carcinoma: a propensity score-matched analysis," Cancer Management and Research, vol. 11, pp. 909-919, 2019.

[53] Y. Zou, J. Zhou, B. Xu, W. Li, and Z. Wang, "Ribonucleotide reductase subunit M2 as a novel target for clear-cell renal cell carcinoma," OncoTargets and Therapy, vol. 12, pp. 32673275, 2019.

[54] Y. Osako, H. Yoshino, T. Sakaguchi et al., "Potential tumor-suppressive role of microRNA-99a-3p in sunitinib-resistant renal cell carcinoma cells through the regulation of RRM2," International Journal of Oncology, vol. 54, no. 5, pp. 1759-1770, 2019.

[55] Z. A. Glaser, H. D. Love, S. Guo et al., “TPX2 as a prognostic indicator and potential therapeutic target in clear cell renal cell carcinoma," Urologic Oncology, vol. 35, no. 5, pp. 286-293, 2017.

[56] L. Yuan, L. Chen, K. Qian et al., "Co-expression network analysis identified six hub genes in association with progression and prognosis in human clear cell renal cell carcinoma (ccRCC)," Genomics Data, vol. 14, pp. 132-140, 2017.

[57] W. Wei, Y. Lv, Z. Gan, Y. Zhang, X. Han, and Z. Xu, "Identification of key genes involved in the metastasis of clear cell renal cell carcinoma," Oncology Letters, vol. 17, no. 5, pp. 4321-4328, 2019. 